\title{
Optimal control of a thermomechanical model of phase transitions in steel
}

\author{
Dietmar Hömberg ${ }^{1 *}$ and Daniela Kern ${ }^{1 * *}$ \\ ${ }^{1}$ WIAS Berlin, Mohrenstr. 39, 10117 Berlin, Germany.
}

\begin{abstract}
We consider an optimal control problem concerning heat treatment of steel. The mathematical model consists of the equations of thermoelasticity coupled to a system of rate laws accounting for the phase transition kinetics. For the coupling a mixture ansatz for the different thermal expansion coefficients is used. In contrast to classical thermoelasticity this leads to residual deformations whenever the resulting phase distribution at end-time is inhomogenous.
\end{abstract}

Copyright line will be provided by the publisher

\section{Problem formulation and existence of solution}

A distinct feature of steel is that one can change its physical properties by thermal interference. The reason for this lies in solid-solid phase transitions actuated by temperature. This is utilized in the heat treatment of steel, which is a process of controlled heating and cooling to achieve a desired distribution of metallurgical phases corresponding to locally varying, heterogeneous physical properties, e.g. hardness or ductility. Such a heat treatment may also lead to sometimes undesired side effects, in particular distortion, i.e. alterations in size and shape of the respective workpiece. In order to describe steel hardening processes including phase induced distortion, we introduce the following variables: let $\sigma$ and $u$ denote stress and displacement respectively, $f$ some internal force (e.g. gravity or zero), $K$ stiffness matrix, $\varepsilon$ strain, $\theta$ temperature, $w$ a volumetric heat source, $z$ a vector of phase fractions, $h$ a function describing the phases' growth rate. Furthermore, the workpiece geometry is given by $\Omega$ with boundary $\Gamma$, which contains $\Gamma_{0}$, the fixed part of the workpiece boundary, and $\Gamma_{1}$, the stress-free part. $q(z)=\delta_{0}+\sum_{i} \delta_{i} z_{i}$ implements a mixture ansatz for coupling phase distribution and displacement by phase dependent expansion.

$$
\begin{array}{rlrl}
-\operatorname{div} \sigma & =f & & \\
\sigma & =K \varepsilon(u)-q(z) \theta I, \\
u & =0 & & \text { on } \Gamma_{0} \\
\nu \sigma & =0 & & \text { on } \Gamma_{1}
\end{array}
$$

$$
\begin{aligned}
\theta_{t}-\Delta \theta+q(z) \operatorname{div}\left(u_{t}\right) & =\mathbf{1} z_{t}+\beta w \\
\theta(0) & =\theta_{0} \\
\frac{d}{d \nu} \theta & =0
\end{aligned}
$$$$
z_{t}=h(\theta, z)
$$$$
z(0)=0
$$

For simplicity, we suppose in the following that $h$ is $C^{2}$ with bounded derivatives.

Solving the phase evolution ODE separately to obtain a dependency $z=z(\theta)$ and utilizing a Galerkin argument, we achieve existence of a solution for the equation system.

Theorem 1.1 For any $w \in L^{2}(Q)$ the state system has a unique solution $(u, \theta, z)$ such that

$$
u \in\left[H^{1}\left(0, T ; H_{\Gamma_{0}}^{1}(\Omega)\right)\right]^{3}, \quad \theta \in H^{2,1}(Q)=L^{2}\left(0, T ; H^{2}(\Omega)\right) \cap H^{1}\left(0, T ; L^{2}(\Omega)\right), \quad z_{j} \in W^{1, \infty}\left(0, T ; L^{\infty}(\Omega)\right) .
$$

$H_{\Gamma_{0}}^{1}(\Omega)$ is a short notation for $\left\{\varphi \in H^{1}(\Omega) ; \varphi=0\right.$ on $\left.\Gamma_{0}\right\}$. The existence result provides a solution operator $S: L^{2}(Q) \rightarrow$ $X, w \mapsto(u, \theta, z)$, for some function space $X$ which we will now specify due to differentiability considerations.

* Dietmar Hömberg E-mail: hoemberg@wias-berlin.de, Phone: +49(0)30 20372491 ,

** Daniela Kern E-mail: kern@wias-berlin.de, Phone: +49(0)30 20372482 ,

applied cooling

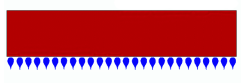

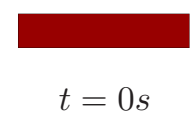

resulting temperature distribution:

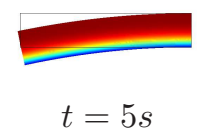

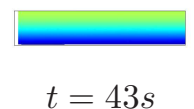

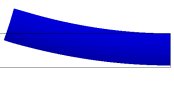

$t=150 \mathrm{~s}$ martensite:

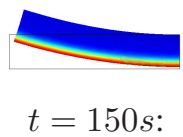

Fig. 1 Cooling of a hot steel slab from below. First a thermoelastic effect takes place that causes the material to shrink at the bottom. But finally martensite formation makes the bulge point the other direction. This deformation converse to the thermoelastic effect is explained by the fact that martensite has a lower density / higher expansion than the other occurring phases. At final time the workpiece has completely cooled down, i.e. is homogeneously at room temperature, and has suffered a lasting deformation. 


\section{Differentiability and optimal control}

Lemma 2.1 Let $\theta \in L^{p}(Q)$ and define $z(\theta)$ as solution to the ODE. Then $z(\theta)$ is twice continuously Fréchetdifferentiable if regarded as an operator $z: L^{p}(Q) \rightarrow W^{1, q}\left(0, T ; L^{q}(\Omega)\right)$, where $q<\frac{p}{2}$.

As $\theta \in H^{2,1}(Q) \hookrightarrow L^{10}(Q) \Rightarrow q<5$, this consideration of $z$ is not too restrictive. Concerning $\psi(z, \theta):=\bar{q} z \theta$ we obtain using Hölder's inequality and the product rule, that $\psi: L^{q}(Q) \times L^{\frac{2 q}{q-2}}(Q) \rightarrow L^{2}(Q)$ is Fréchet-differentiable and $(\bar{q} z \theta)^{\prime} h=\bar{q} z^{\prime} h \theta+\bar{q} z \theta^{\prime} h$. Applying the same argumentation twice, we achieve that $\bar{q} z \theta$ is twice continuously Fréchetdifferentiable. The Implicit Function Theorem for Banach spaces then yields

Theorem 2.2 The solution operator $S$ is twice continuously Fréchet-differentiable. $S^{\prime}(w) h=(\eta, \lambda, \zeta)^{T}$ and $S^{\prime \prime}(w)\left[h_{1}, h_{2}\right]=(\mu, \vartheta, \xi)^{T}$ are the solutions to the respective linearized equations.

We are now prepared to consider the following optimal control problem:

\section{Problem 2.3}

$$
\begin{gathered}
\text { Minimize } J(w)=\int_{0}^{T} \int_{\Omega} L_{1}(u, \theta) d x d t+\int_{\Omega} L_{2}(z(T)) d x+\frac{\alpha}{2} \int_{0}^{T} \int_{\Omega} w^{2} d x d t \\
\text { subject to } \int_{\Omega}(K \varepsilon(u)-q(z) \theta I): \varepsilon(v) d x=\int_{\Omega} f v d x \quad \forall v \in\left[H_{\Gamma_{0}}^{1}(\Omega)\right]^{3} \\
\int_{\Omega} \theta_{t} \varphi d x+\int_{\Omega} \nabla \theta \nabla \varphi d x=\int_{\Omega}(1 h(\theta, z)+\beta w) \varphi d x \quad \forall \varphi \in H^{1}(\Omega) \\
z_{t}=h(\theta, z) \quad \text { in } \Omega \times(0, T) \\
\theta(0)=\theta_{0}, \quad z(0)=0 \quad \text { in } \Omega
\end{gathered}
$$

and $g \in U_{a d} \subset L^{2}(Q)$, e.g. $U_{a d}=\left\{w \in L^{2}(Q) ; w_{a}(x, t) \leq w(x, t) \leq w_{b}(x, t) \forall(x, t) \in Q\right\}, w_{a}, w_{b} \in L^{2}(Q)$.

Utilizing a compactness argument we obtain the existence of an optimal control.

Theorem 2.4 Every locally optimal control $\bar{w}$ in $U_{\text {ad }}$ of 2.3 with $S(\bar{w})=(\bar{u}, \bar{\theta}, \bar{z})$ and adjoint state $(\mu, \vartheta, \zeta)$ given by the adjoint system

$$
\begin{array}{rlrl}
\int_{\Omega} K \varepsilon(\mu): \varepsilon(v) d x-\int_{\Omega} \frac{\partial}{\partial u} L_{1}(\bar{u}, \bar{\theta}) v d x & =0 & \forall v \in\left[H_{\Gamma_{0}}^{1}(\Omega)\right]^{3} \\
-\vartheta_{t}-q(\bar{z}) \operatorname{div} \mu-\Delta \vartheta-h_{\theta}(\bar{\theta}, \bar{z})(\mathbf{1} \vartheta+\zeta)-\frac{\partial}{\partial \theta} L_{1}(\bar{u}, \bar{\theta}) & =0 & & \text { in }(0, T) \times \Omega \\
-\zeta_{t}-h_{z}(\bar{\theta}, \bar{z})^{T}(\mathbf{1} \vartheta+\zeta)+\bar{q} \bar{\theta} \operatorname{div} \mu & =0 & & \text { in }(0, T) \times \Omega \\
\frac{d}{d \nu} \vartheta=0 & \text { on }(0, T) \times \partial \Omega \\
\vartheta(T)=0, \quad \zeta(T)=\frac{\partial}{\partial z} L_{2}(\bar{z}(T)) & \text { in } \Omega
\end{array}
$$

satisfies the variational inequality $\int_{0}^{T} \int_{\Omega}(\alpha \bar{w}-\beta \vartheta)(w-\bar{w}) d x d t \geq 0 \quad \forall w \in U_{a d}$.

Exploiting the differentiability properties stated above and applying standard theory, also a formulation of second order conditions can be given.

\section{Outlook and conclusion}

We chose a model to represent the interdependence of temperature, metallurgical phases, and mechanical quantities like stress and displacement. It was possible to conduct an exhausting analysis of the according optimal control problem. It remains our goal to extend the model by a stronger coupling but preserving important mathematical properties, in particular sufficient differentiability of the solution operator. This is due to the goal to finally implement a second order numerical method and apply it to problems arising in engineering.

Acknowledgements This work was partially supported by DFG Research Center Matheon, project C11: Modeling and optimization of phase transitions in steel.

\section{References}

[1] F. Tröltzsch Optimale Steuerung partieller Differentialgleichungen. Vieweg Verlag 2005

[2] C. Chełminski, D. Hömberg, D. Kern On a thermomechanical model of phase transitions in steel. Matheon preprint 387 